\title{
LETTER
}

Chronic lymphocytic leukemia

\section{Anti-SARS-CoV-2 antibody response in patients with chronic lymphocytic leukemia}

\author{
Lindsey E. Roeker $\mathbb{D}^{1} \cdot$ David A. Knorr $\mathbb{D}^{1} \cdot$ Melissa S. Pessin $\mathbb{1}^{2} \cdot$ Lakshmi V. Ramanathan $^{2} \cdot$ Meghan C. Thompson ${ }^{1}$. \\ Lori A. Leslie ${ }^{3} \cdot$ Andrew D. Zelenetz $^{4} \cdot$ Anthony R. Mato $^{1}$
}

Received: 27 July 2020 / Revised: 1 August 2020 / Accepted: 12 August 2020 / Published online: 27 August 2020

(c) The Author(s), under exclusive licence to Springer Nature Limited 2020

\section{To the Editor:}

Characterizing antibody $(\mathrm{Ab})$-mediated immune response to SARS-CoV-2 is fundamental to understanding COVID-19 epidemiology, reinfection potential, and vaccine development, particularly in immunocompromised patients. The first report of SARS-CoV-2 directed Abs demonstrated that $100 \%$ of 34 hospitalized patients developed virus-specific IgM and IgG by 3 weeks following symptom onset with rising IgG titers during weeks 4 through 7 (end of study period) [1]. A subsequent series of 285 patients with COVID-19 demonstrated that $100 \%$ developed virusspecific IgG 17-19 days after symptom onset [2]. While studies with short follow-up have demonstrated that lower proportions of patients develop anti-SARS-CoV-2 IgG $[3,4]$, studies with follow-up extending into the period of expected peak IgG levels have confirmed high rates of antiSARS-CoV-2 IgG development (98-100\%; peak day 30) [5-7]. Persistence of $\mathrm{Ab}$ responses requires further study. Given genomic similarity between SARS-CoV-2 and SARS-CoV, dynamics of SARS-CoV Ab response may be relevant; IgG peaked between months 2 and 4 and declined thereafter, though antigen-specific IgG was persistently detected up to 24 months [8,9]. Data of this nature are not yet available for SARS-CoV-2. Much remains unknown

Lindsey E. Roeker

roekerl@mskcc.org

1 Leukemia Service, Department of Medicine, Memorial Sloan Kettering Cancer Center, New York, NY, USA

2 Department of Laboratory Medicine, Memorial Sloan Kettering Cancer Center, New York, NY, USA

3 John Theurer Cancer Center at Hackensack University Medical Center, Hackensack, NJ, USA

4 Lymphoma Service, Department of Medicine, Memorial Sloan Kettering Cancer Center, New York, NY, USA about neutralizing capacity of $\mathrm{Ab}$ and clinical relevance of $\mathrm{Ab}$ responses for immune status.

Notably, most data on serologic response to SARS-CoV-2 have come from immunocompetent adults. In contrast, a small series of ten cancer patients (mixed histology) showed a seroconversion rate of $30 \%(3 / 10)$ at 15 days following documented positive RT-PCR, though data on seroconversion at later timepoints were not available [10]. Notably, of the seven seronegative patients with PCR-based COVID-19 diagnosis, six had received cytotoxic therapy or major surgery in the preceding 4 weeks [10].

$\mathrm{Ab}$ responses in patients with chronic lymphocytic leukemia (CLL) are of particular interest given cellular and humoral immunodeficiencies resulting from the disease and CLL-directed therapies [11]. While robust epidemiologic data regarding risk of SARS-CoV-2 infection are not yet available, case fatality rates in series of CLL patients with symptomatic COVID-19 are 30-33\% [12, 13]. Furthermore, studies of seroconversion in response to vaccines in patients with CLL have demonstrated suboptimal responses [14]. Thus, patients with CLL are at risk for severe COVID-19 given their underlying immunodeficiency, often advanced age, and potentially suboptimal serologic response to infection or related vaccine.

To characterize immune response to COVID-19 in patients with CLL, we identified all patients who receive care for an antecedent diagnosis of CLL at Memorial Sloan Kettering Cancer Center diagnosed with COVID-19 via PCR for SARS-CoV-2 RNA from a nasopharyngeal swab between March 18 and April 29, 2020. We retrospectively examined patients subsequently tested for anti-SARS-CoV-2 IgG Ab utilizing the Abbott Architect SARS-CoV-2 IgG assay (Abbott Park, IL, USA) to detect serum IgG Abs directed against the nucleocapsid protein of SARS-CoV-2 in routine clinical practice. We examined baseline characteristics, CLLdirected therapy history, and details regarding COVID-19 testing and clinical course. Patients were defined as having severe COVID-19 if they required supplemental oxygen and/ 
or ICU admission. Immunoglobulin levels were examined within 3 months of COVID-19 diagnosis; hypogammaglobulinemia was defined as $\mathrm{IgG}<650 \mathrm{mg} / \mathrm{dL}$. As a preliminary, hypothesis generating analysis, we used logistic regression to assess association between hypogammaglobulinemia and CLL-directed therapy (current therapy vs. observation, current BTK inhibitor (BTKi) therapy) and Ab development. Proportions were compared with Fisher's exact test.

We identified 30 consecutive patients at our center with CLL who tested positive for SARS-CoV-2 RNA, either in the setting of symptomatic COVID-19 or through screening at entry to the medical system. Hospital admission was required for $63 \%, 50 \%$ had severe COVID-19, and case fatality rate was $13 \%$ in this cohort. Two patients $(7 \%)$ had been discharged from their COVID-19 hospital course with hospice care; both remain alive at the time of analysis. Baseline characteristics, CLL treatment history, details regarding COVID-19 course are included in Table 1.

Twenty-one patients were subsequently tested for antiSARS-CoV-2 IgG Abs; the four patients who died due to COVID-19 and/or its complications in this cohort did not have serology testing prior to death, and testing was not performed for an additional five patients following acute infection. For the subset of patients who underwent IgG testing, serology testing occurred at a median of 55 days (range 28-93 days) following PCR diagnosis.

Table 1 Baseline characteristics, lab characterization at time of serology testing, and CLL-directed therapy history.

\begin{tabular}{|c|c|}
\hline Characteristic & $\begin{array}{l}\text { Proportion, unless } \\
\text { otherwise specified } \\
(n=30)\end{array}$ \\
\hline \multicolumn{2}{|l|}{ Baseline characteristics } \\
\hline $\begin{array}{l}\text { Age at COVID-19 diagnosis, } \\
\text { median (range) }\end{array}$ & $65(41-82)$ \\
\hline $\begin{array}{l}\text { Age at CLL diagnosis, median } \\
\text { (range) }\end{array}$ & $51(35-76)$ \\
\hline Male & $73 \%$ \\
\hline \multicolumn{2}{|l|}{ CLL-directed therapy history } \\
\hline Never treated & $47 \%$ \\
\hline Current observation & $67 \%$ \\
\hline Current therapy & $33 \%$ \\
\hline Current BTKi & $20 \%$ \\
\hline Prior anti-CD20 monoclonal $\mathrm{Ab}$ & $43 \%$ \\
\hline Prior fludarabine or bendamustine & $30 \%$ \\
\hline Prior cellular immunotherapy & $3 \%$ \\
\hline \multicolumn{2}{|l|}{ COVID-19 course } \\
\hline Required hospital admission & $63 \%$ \\
\hline Required ICU admission & $36 \%$ \\
\hline Required oxygen & $50 \%$ \\
\hline Required intubation & $23 \%$ \\
\hline Death & $13 \%$ \\
\hline
\end{tabular}

Fourteen of $21(67 \%)$ patients tested positive for antiSARS-CoV-2 Abs, while 7 of 21 (33\%) did not have detectable Abs. Testing occurred at median of 57 (range 28-93) vs. 51 (range 38-62) days for patients with positive vs. negative anti-SARS-CoV-2 IgG. Three of 14 (21\%) who tested positive for anti-SARS-CoV-2 IgG were receiving CLL-directed therapy at the time of COVID-19 diagnosis (1 with venetoclax, 1 with ibrutinib, and 1 with bendamustine/ obinutuzumab). Four of seven (57\%) who did not develop anti-SARS-CoV-2 IgG were receiving CLL-directed therapy (three with ibrutinib, one with venetoclax/obinutuzumab).

Of the patients who had serologic testing, $44 \%(7 / 16)$ had hypogammaglobulinemia, 50\% (7/14) had $\operatorname{IgM}<50$ $\mathrm{mg} / \mathrm{dL}$, and $14 \%(2 / 14)$ had $\operatorname{IgA}<40 \mathrm{mg} / \mathrm{dL}$. Hypogammaglobulinemia (OR 0.05, 95\% CI 0.003-0.7, $p=$ 0.027 ) was negatively associated with development of antiSARS-CoV-2 IgG. Currently receiving CLL-directed therapy (OR $0.2,95 \%$ CI $0.03-1.5, p=0.11$ ) or BTKi (OR 0.1, $95 \%$ CI $0.008-1.3, p=0.08$ ) were not significantly associated with anti-SARS-CoV-2 IgG development. Of patients with severe COVID-19, 78\% had positive antiSARS-CoV-2 IgG while 58\% with nonsevere COVID-19 were positive for anti-SARS-CoV-2 $\operatorname{IgG}(p=0.64)$.

While published data on anti-SARS-CoV-2 IgG response suggest high (98-100\%) rates of positive $\mathrm{IgG}$ by 17-19 days after PCR-based diagnosis, this series suggests that CLL patients have a lower rate of anti-SARS-CoV-2 IgG development (67\%). Hypogammaglobulinemia was negatively associated with anti-SARS-CoV-2 IgG development. Trends toward lack of anti-SARS-CoV-2 IgG development for those currently receiving CLL-directed therapy or BTKi did not reach statistical significance in this small cohort. There was no significant difference in rates of $\mathrm{Ab}$ development between those with severe vs. nonsevere COVID-19. These findings need to be interpreted with caution and explored in larger cohorts. Notably, this study did not include patients who died because of COVID-19. Thus, we cannot draw conclusions about $\mathrm{Ab}$ formation in CLL patients with severe COVID-19 resulting in death or how lack of testing in this population affected the proportion of patients with positive anti-SARS-CoV-2 IgG.

These hypothesis generating data have important implications as we aim to understand CLL patients' immune response to infection or potential vaccination. Patients in this series were tested 28-93 days following PCR-based diagnosis. We cannot exclude the possibility that these patients had transient immune responses not captured by the testing window. However, these data suggest that $33 \%$ of tested CLL patients did not have a persistent $\mathrm{Ab}$-mediated response, which may have implications for risk of subsequent infection. As patients with CLL appear to have variable humoral immune response to infection, it could be hypothesized that some CLL patients may have an impaired response to 
potential vaccines that rely on $\mathrm{Ab}$ production to confer immunity [15]. Should these findings be validated, response to candidate vaccines will need to be specifically examined in immunocompromised populations. Further characterization of cell-mediated immune response and effect of CLL-directed therapy on immune response are ongoing.

Acknowledgements This research was supported in part by the NIH/ NCI Cancer Center Support Grant P30 CA008748, NCI 1K08CA248966-01, and the National Center for Advancing Translational Sciences through Rockefeller University grants UL1TR001866 and KL2TR001865 NIH Clinical and Translational Science Award Program. LER and DAK recognize support from the American Society of Hematology Research Training Award for Fellows outside of the submitted work.

\section{Compliance with ethical standards}

Conflict of interest ER has received grant funding from American Society of Hematology outside of the submitted work and has minority ownership interest in AbbVie and Abbott Laboratories; LVR has received institutional research funding outside of submitted work from Abbott, IBM, and BioPortal; LAL is a member of speaker's bureau for Seattle Genetics, Celgene/BMS, KitePharma, BeiGene, Pharmacyclics/Janssen, AstraZeneca, Epizyme, Karyopharm, advisory board participant for Bayer, Seattle genetics, ADC therapeutics, Abbvie, Janssen, Pharmacyclics, Kite, AstraZeneca, TG Therapeutics; ADZ has received research grants from Abbvie, Adaptive Biotechnologies, BMS, BeiGene, Genentech/Roche, MEI Pharma, consulting fees from Amgen, AstraZeneca, BeiGene, Genentech/Roche, Janssen, JUNO/ Celgene/BMS, Kite/Gilead, MEI Pharma, Pfizer, Pharmacyclics, Sandoz/Novartis, and serves on the scientific advisory board of Adaptive Biotechnologies, Lymphoma Research Foundation; ARM has received grants, personal fees and other from TG Therapeutics, grants and personal fees from Pharmacyclics, grants and personal fees from Janssen, grants and other from Celgene, grants and personal fees from Genentch, grants and personal fees from Abbvie, grants and personal fees from Adaptive, grants from Loxo, grants from Sunesis, grants from Regeneron, grants from DTRM, personal fees from Beigene, grants and personal fees from AstraZenica.

Publisher's note Springer Nature remains neutral with regard to jurisdictional claims in published maps and institutional affiliations.

\section{References}

1. Xiao AT, Gao C, Zhang S. Profile of specific antibodies to SARSCoV-2: the first report. J Infect. 2020;81:147-78.
2. Long QX, Liu BZ, Deng HJ, Wu GC, Deng K, Chen YK, et al. Antibody responses to SARS-CoV-2 in patients with COVID-19. Nat Med. 2020;26:845-8.

3. Zhao J, Yuan Q, Wang H, Liu W, Liao X, Su Y, et al. Antibody responses to SARS-CoV-2 in patients of novel coronavirus disease 2019. Clin Infect Dis. 2020. https://doi.org/10.1093/cid/ciaa 344.

4. Guo L, Ren L, Yang S, Xiao M, Chang, Yang F, et al. Profiling early humoral response to diagnose novel coronavirus disease (COVID-19). Clin Infect Dis. 2020;71:778-85.

5. Du Z, Zhu F, Guo F, Yang B, Wang T. Detection of antibodies against SARS-CoV-2 in patients with COVID-19. J Med Virol. 2020. https://doi.org/10.1002/jmv.25820.

6. Qu J, Wu C, Li X, Zhang G, Jiang Z, Li X, et al. Profile of IgG and $\operatorname{IgM}$ antibodies against severe acute respiratory syndrome coronavirus 2 (SARS-CoV-2). Clin Infect Dis. 2020. https://doi. org/10.1093/cid/ciaa489.

7. Bryan A, Pepper G, Wener MH, Fink SL, Morishima C, Chaudhary A, et al. Performance characteristics of the Abbott architect SARS-CoV-2 IgG assay and seroprevalence in Boise, Idaho. J Clin Microbiol. 2020;58:e00941-20.

8. Lin Q, Zhu L, Ni Z, Meng H, You L. Duration of serum neutralizing antibodies for SARS-CoV-2: lessons from SARS-CoV infection. J Microbiol Immunol Infect. 2020. https://doi.org/10. 1016/j.jmii.2020.03.015.

9. Mo H, Zeng $\mathrm{G}$, Ren $\mathrm{X}$, Li $\mathrm{H}, \mathrm{Ke} \mathrm{C}$, Tan $\mathrm{Y}$, et al. Longitudinal profile of antibodies against SARS-coronavirus in SARS patients and their clinical significance. Respirology. 2006;11:49-53.

10. Solodky ML, Galvez C, Russias B, Detourbet P, N'Guyen-Bonin $\mathrm{V}$, Herr $\mathrm{AL}$, et al. Lower detection rates of SARS-COV2 antibodies in cancer patients versus health care workers after symptomatic COVID-19. Ann Oncol. 2020. https://doi.org/10.1016/j. annonc.2020.04.475.

11. Forconi F, Moss P. Perturbation of the normal immune system in patients with CLL. Blood. 2015;126:573-81.

12. Scarfo L, Chatzikonstantinou T, Rigolin GM, Quaresmini G, Motta M, Vitale C, et al. COVID-19 severity and mortality in patients with chronic lymphocytic leukemia: a joint study by ERIC, the European Research Initiative on CLL, and CLL Campus. Leukemia. 2020. https://doi.org/10.1038/s41375-020-0959-x: $1-10$.

13. Mato AR, Roeker LE, Lamanna N, Allan J, Leslie LA, Pagel JM, et al. Outcomes of COVID-19 in patients with CLL: a multicenter, international experience. Blood. 2020. https://doi.org/10.1182/ blood.2020006965.

14. Sinisalo M, Aittoniemi J, Kayhty H, Vilpo J. Vaccination against infections in chronic lymphocytic leukemia. Leuk Lymphoma 2003;44:649-52.

15. Jackson LA, Anderson EJ, Rouphael NG, Roberts PC, Makhene $\mathrm{M}$, Coler RN, et al. An mRNA vaccine against SARS-CoV-2preliminary report. N Engl J Med. 2020. https://doi.org/10.1056/ NEJMoa2022483. 